\title{
COMPARISONS OF PARTICLE TRACKING AND CHARGE DEPOSITION SCHEMES FOR A FINITE ELEMENT GUN CODE*
}

\author{
E. M. Nelson $^{\dagger}$, LANL, Los Alamos, NM; K. R. Eppley, J. J. Petillo, SAIC, Boston, MA \\ S. J. Humphries, Jr., Field Precision, Albuquerque, NM
}

\begin{abstract}
A new finite element gun code is under development. In an effort to improve the gun code model, a concept[1] has been proposed recently that treats fields in a typical way, but includes a unique, formal approach to both particle tracking and source allocation. Being a new approach, there are concerns about the speed, accuracy, and appropriateness of this proposal for the electrostatic, steady-state particle-in-cell (PIC) gun model. In order to resolve some of these issues, a variety of particle tracking and charge deposition schemes are being evaluated with respect to accuracy, speed, robustness, and effect on the model. This includes various methods for computing the electric field at the particle locations. For this study, we are using the SAIC 3D gun code AVGUN as a testbed to incorporate and evaluate these methods. Results of a theoretical analysis of the methods will be presented, and a comparison will be made with the empirical results.
\end{abstract}

\section{INTRODUCTION}

Some concepts for a new finite element (FE) gun code were proposed recently[1]. This new FE gun code will model complicated 3D problems. The proposed concepts focused on dealing effectively with issues a gun code will encounter on an unstructured grid.

For example, rays are traced through the unstructured grid element by element using local coordinates in each element. Such a particle-tracking scheme has numerous qualities which we will not enumerate here. Concerning the finite element field calculation, the potential is a linear combination of basis functions $N_{i}(\mathbf{x})$,

$$
\phi(\mathbf{x})=\sum_{i=1}^{n} \alpha_{i} N_{i}(\mathbf{x}) .
$$

The electric field is the derivative of this potential with no additional smoothing,

$$
\mathbf{E}(\mathbf{x})=-\nabla \phi(\mathbf{x})=-\sum_{i=1}^{n} \alpha_{i} \nabla N_{i}(\mathbf{x})
$$

The charge deposition scheme follows from the formal application of the finite element formulation: the linear

\footnotetext{
* Work supported by DOE, contract W-7405-ENG-36, and ONR, NRL contract N 00014-97-C-2076.

† Email: enelson@lanl.gov
}

charge density multiplied by the basis functions are integrated along the ray trajectories,

$$
b_{i}=\int_{\Omega} \rho N_{i} d \Omega=\sum_{j=1}^{n_{r}} \int_{R_{j}} \frac{I_{j}}{v_{j}(s)} N_{i}\left(\mathbf{x}_{j}(s)\right) d s .
$$

There are numerous questions concerning the speed, accuracy and utility of the proposed concepts. In this paper we compare and contrast three charge deposition schemes and three electric field interpolation schemes in the context of a simple 1D problem which can be solved analytically. The basis functions $N_{i}$ for $\phi$ are linear. The charge deposition schemes studied are constant (where the charge in an element is shared equally among the element's nodes), nearest grid point (NGP) and linear (i.e., Eq. 3). The field interpolation schemes are constant (i.e., Eq. 2) and two forms of linear interpolation discussed below.

\section{THE TEST CASE}

We studied a simple 1D diode problem which can be solved analytically. The parameters of the problem are listed in Table 1. A finite particle energy at the cathode was chosen so that the singularity at a space charge limited (SCL) cathode is avoided. Similarly, the current transported across the diode was fixed. Differences in SCL emission algorithms in various codes do not affect this study. The limiting case of perfect integration of the equations of motion in the presence of the discretized fields was pursued, so issues arising from finite integration steps are mitigated. The voltages were chosen so that the problem is essentially nonrelativistic. The meshes are uniform.

\section{Table 1: 1D Diode Test Case Parameters}

\begin{tabular}{|l|l|}
\hline anode-cathode gap, $d$ & $0.05 \mathrm{~m}$ \\
anode-cathode voltage, $\phi_{d}-\phi_{0}$ & $4.5 \mathrm{kV}$ \\
electron energy at cathode, $\phi_{0}$ & $10 \mathrm{eV}$ \\
current density, $J$ & $-250 \mathrm{~A} / \mathrm{cm}^{2}$ \\
\hline cathode electric field, $E(0)$ & $-34.9187 \mathrm{kV} / \mathrm{m}$ \\
anode electric field, $E(d)$ & $-115.782 \mathrm{kV} / \mathrm{m}$ \\
transit time, $\tau$ & $2.86391 \mathrm{~ns}$ \\
\hline
\end{tabular}

For this 1D problem there are only two measures of the quality of the beam calculation: transit time and particle energy. The computational schemes presented in this paper 
all conserve energy precisely at the anode. That is, the particle energy will be exactly the anode voltage, regardless of the discretization of the anode-cathode gap. The transit time is not exact in any of the schemes studied here, so transit time error will be our main measure of the quality of the beam calculation. The analytic results in Table 1 were computed with a non-relativistic model.

The quality of the field calculation can be measured by the error of the computed potential at the nodes. Alternatively, the transit time error can be considered an integral measure of the error in the field calculation. The transit time is

$$
\tau=\int_{0}^{d} \frac{d x}{v(x)}=\int_{0}^{d} \sqrt{\frac{-m}{2 q \phi_{e}(x)}} d x
$$

where $-q \phi_{e}(x)$ is the kinetic energy of the particle. The potential $\phi_{e}$ derives from the integration of the electric field,

$$
\phi_{e}(x)=\phi_{0}-\int_{0}^{x} E\left(x^{\prime}\right) d x^{\prime} .
$$

In the analytic case there is no distinction between $\phi_{e}$ and $\phi$ (we take the arbitrary constant difference to be zero), but in the discretized problem the field interpolation algorithm may introduce a distinction. The transit time error is

$$
\begin{aligned}
\tau_{h}-\tau & =\int_{0}^{d} \sqrt{\frac{-m}{2 q \phi_{e}(x)}}-\sqrt{\frac{-m}{2 q \phi(x)}} d x \\
& \cong \int_{0}^{d} \sqrt{\frac{-m}{8 q \phi^{3}(x)}}\left(\phi_{e}(x)-\phi(x)\right) d x
\end{aligned}
$$

\section{TESTS WITH AVGUN}

AVGUN is a $3 \mathrm{D}$ gun code which employs a Cartesian mesh. For this study, the code was modified to allow both linear and constant charge deposition schemes, and both linear and constant field interpolation schemes. In AVGUN, the linear field interpolation is performed on a dual grid, as is typical for finite difference (FD) codes. The electric field on the half dual cells at the ends of the grid is constant. An FE field solution option was added to AVGUN's existing FD field solver.

The details of the matrix equation representing Poisson's equation in 3D are different between the FD and FE algorithms, but for a $1 \mathrm{D}$ problem the matrices are equivalent. The 1D test problem was modeled with AVGUN using both algorithms, and the FE case agrees with the FD case.

The quality of the AVGUN calculations was measured by the error in the electric field at the cathode. This electric field was computed by linearly extrapolating the electric fields in the first two cells $(E(h / 2)$ and $E(3 h / 2))$ to the cathode. The integration step size $h_{\text {ray }}$ was varied, and the results were linearly extrapolated to infinitesimal step size. The dependence of the relative error on integration step size was approximately $(600 \%) h_{\text {ray }} / d$. The results are shown in Fig. 1. From this test, it appears that the constant charge deposition scheme performs better than the linear charge

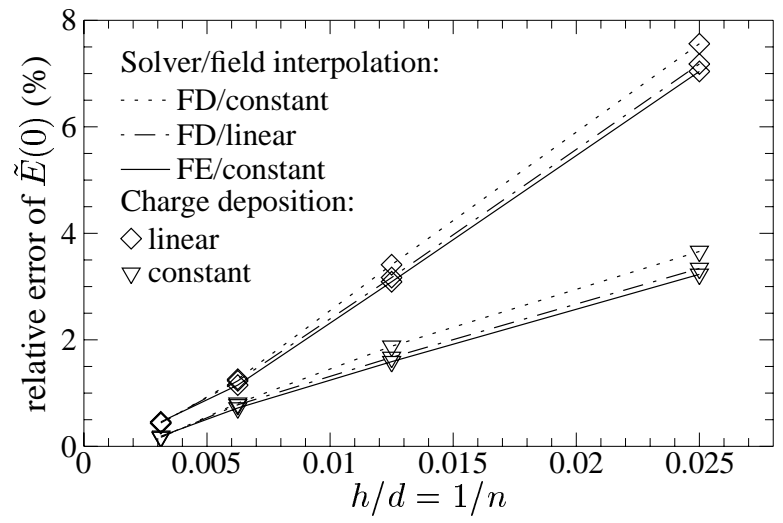

Figure 1: Relative error of the electric field $\tilde{E}(0)$ at the cathode, computed by linear extrapolation of the electric field in the first two cells, versus cell size $h$.

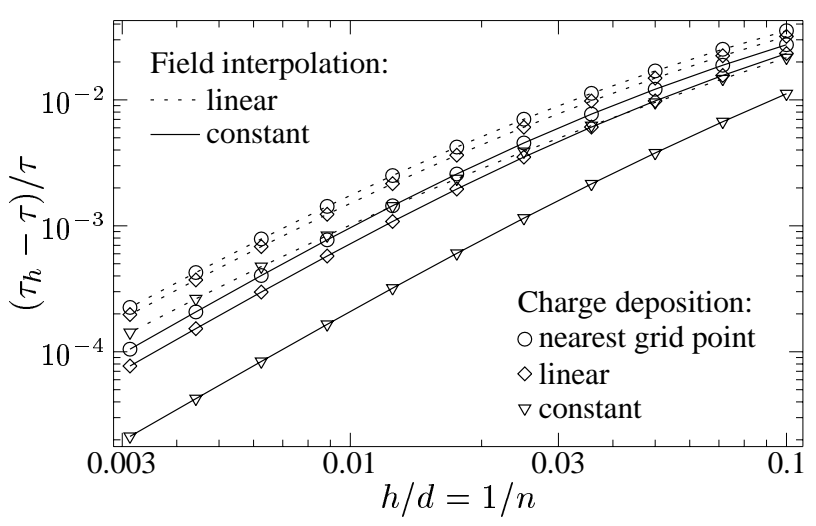

Figure 2: Relative error of the transit time $\tau_{h}$ versus cell size $h$, using various field interpolation and charge deposition schemes.

deposition scheme. Furthermore, the choice of field interpolation scheme shows no significant effect.

Transit time in the AVGUN simulations was also scrutinized, but the results were difficult to interpret due to additional random errors, perhaps residual errors left by the iterative solvers, which were about the same size as the transit time errors of interest.

\section{TESTS WITH A 1D CODE}

Further tests were performed with small 1D codes that integrated the equations of motion cell by cell, either analytically or with a high order adaptive integrator. Furthermore, the matrix equations were solved directly instead of iteratively, and the relaxation procedure was performed to the limit dictated by numerical precision. The results are shown in Figs. 2-4. Linear field interpolation in this case means an average electric field is computed at each node, and then these average electric fields are interpolated linearly within each cell. There is no dual grid.

The transit time errors shown in Fig. 2 would indicate once again that the constant charge deposition scheme was superior. These two results were surprising to the authors, 


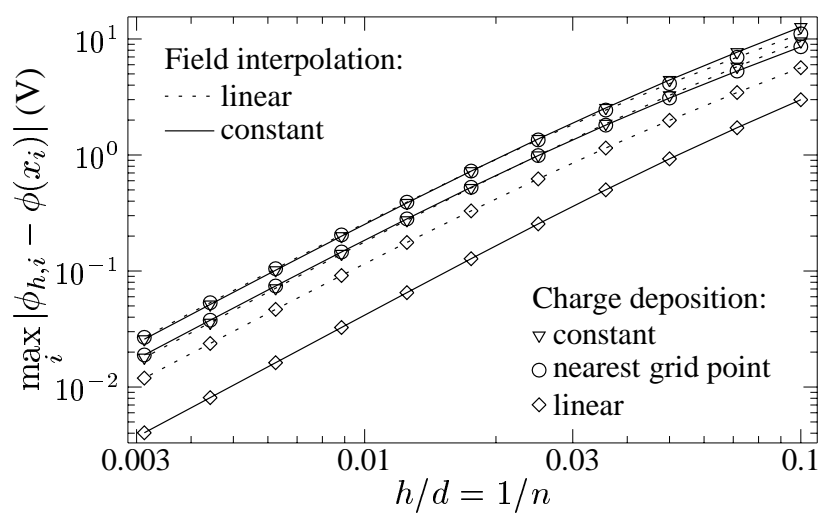

Figure 3: Maximum error of the nodal potentials $\phi_{h, i}$ versus cell size $h$.

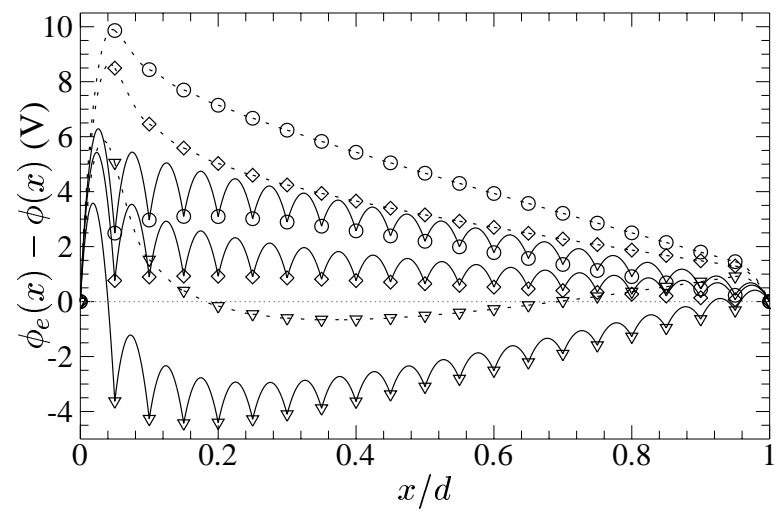

Figure 4: Potential error $\phi_{e}(x)-\phi(x)$ for $h / d=1 / 20$ and various field interpolation and charge deposition schemes. Symbols identify the potential error at nodes. See Fig. 3 for legend.

so further explanation was pursued. In contrast, looking at the error in the potential at the nodes, shown in Fig. 3, one would conclude that the linear charge deposition, constant field interpolation scheme is superior.

Detailed examination of the potential reveals the source of this apparent discrepancy. While the potential at the nodes is very good in the linear charge deposition, constant field interpolation scheme, the error in the potential is dominated by the limited ability of the linear basis functions to interpolate the exact potential. The large bumps in Fig. 4 illustrate this. Error analysis of the finite element method $[2,3]$ also suggests this-the electric field error is minimized directly. Minimization of the potential error is secondary. Referring to Eq. 7, one can see that the transit time error arises mainly from the first few "interpolation error" bumps, where the particles are slowest and the relative velocity error is largest.

The dependence of the transit time error on the charge deposition schemes can now be understood. The constant scheme deposits more charge in the gap (in the sense that the components $b_{i}$ of the source vector are larger), so the potential is depressed, and the transit time error due to interpolation error is partially cancelled. The NGP scheme deposits less charge in the gap, so the potential is increased, adding to the interpolation error and thus the transit time error.

This situation does not depend on the particular parameters of the 1D test problem. Choosing a case with a smoother charge distribution (e.g., by injecting the beam at a higher energy) reduces the magnitude of the error, but the constant charge deposition scheme will still give a better transit time than the linear charge deposition scheme.

Fig. 4 suggests that an electric field calculation scheme which better interpolates the potential at the nodes would significantly reduce the transit time error. The linear field interpolation schemes studied here are defective in the sense that $\phi_{e}$ from Eq. 5 does not agree with the potential $\phi_{h, i}$ at the nodes. The potentials $\phi_{e}$ for these cases are systematically higher than the corresponding constant field interpolation cases, as shown in Fig. 4. This difference is mainly due to the field interpolation-the change in the nodal potentials is relatively modest in comparison. This difference in $\phi_{e}$ increases the transit time error.

Some existing FE gun codes (e.g., DEMEOS[4] and TRAK[5]) fit the potential at nearby nodes to a quadratic function, and then base the electric field on derivatives of this fit potential. A similar scheme which ensures agreement of the fit potential at the cell's nodes could make the linear charge deposition scheme unambiguously superior. Likewise, a higher order (e.g., quadratic) basis for the potential would significantly reduce the interpolation error and at the same time significantly reduce the error at the nodes.

\section{CONCLUSION}

A study of three charge deposition schemes and three field interpolation schemes in the context of a simple 1D problem indicates that the constant charge deposition, constant field interpolation scheme provides for the best simulation of the beam. However, the results also suggest that the path to further improvement of a linear basis FE gun code involves linear charge deposition combined with a better field interpolation scheme.

\section{REFERENCES}

[1] Eric M. Nelson and John J. Petillo, "Conceptual Description of a Novel Finite Element Gun Code," ICAP'98.

[2] Claes Johnson, "Numerical Solution of Partial Differential Equations by the Finite Element Method," Cambridge Univ. Press, 1987.

[3] Strang and Fix, "An Analysis of the Finite Element Method," Prentice-Hall, 1973.

[4] R. True, "A General Purpose Relativistic Beam Dynamics Code," CAP'93.

[5] S. Humphries Jr., "Finite-element Methods for Electron Gun Design,” ICOPS'96. 